\title{
Adaptive Control Theory and Applications
}

\author{
Chengyu Cao, ${ }^{1}$ Lili $\mathrm{Ma},{ }^{2}$ and Yunjun $\mathrm{Xu}^{3}$ \\ ${ }^{1}$ Department of Mechanical Engineering, University of Connecticut, Storrs, CT 06269-4077, USA \\ ${ }^{2}$ Electronics and Mechanical Department, Wentworth Institute of Technology, Boston, MA 02115, USA \\ ${ }^{3}$ Department of Mechanical, Materials, and Aerospace Engineering, University of Central Florida, Orlando, FL 32816, USA
}

Correspondence should be addressed to Chengyu Cao, ccao@engr.uconn.edu

Received 15 August 2012; Accepted 15 August 2012

Copyright (C) 2012 Chengyu Cao et al. This is an open access article distributed under the Creative Commons Attribution License, which permits unrestricted use, distribution, and reproduction in any medium, provided the original work is properly cited.

Adaptive control is an active field in the design of control systems to deal with uncertainties. The key difference between adaptive controllers and linear controllers is the adaptive controller's ability to adjust itself to handle unknown model uncertainties. Adaptive control is roughly divided into two categories: direct and indirect. Indirect methods estimate the parameters in the plant and further use the estimated model information to adjust the controller. Direct methods are ones wherein the estimated parameters are those directly used in the adaptive controller.

Recently, much effort has been placed in adaptive control in both theory and applications. Theory-wise, new controller design techniques are introduced to handle nonlinear and time-varying uncertainties. Broader systems with larger nonlinear uncertainties can be covered by these developments. As a result, adaptive control finds use in various real world applications. This special session generalizes some of the latest results of adaptive control in both theory and applications. After a thorough review process, 8 papers were selected. The papers in this special section include the following.

The paper entitled "Adaptive control for nonlinear systems with time-varying control gain" by A. Rincon and F. Angulo, a scheme for nonlinear plants with time-varying control gains and time-varying plant coefficients is proposed and applied on a plant model consisting of a Brunovsky type model with polynomials as approximators. The methodology has been applied to the speed control of a permanent magnet synchronous motor (PMSM) and proper tracking results have been achieved.

The paper entitled "Adaptive impedance control to enhance human skill on a haptic interface system" by S. Suzuki and
K. Furuta, adaptive assistive control for a haptic interface system is proposed. An adaptive mechanism derived from a Lyapunov candidate function is used to tune an impedance of the virtual model for the haptic device according to the identified operator's characteristics for enhanced performance. It was verified that the operator's characteristics can be estimated and further enhanced.

The paper entitled "Pilot-induced oscillation suppression by using L1 adaptive control" by C. Wang and C. Cao, where pilot-induced oscillation (PIO) is a phenomenon that occurs in both flight tests and operational aircrafts. In this paper, the $L 1$ adaptive controller has been introduced to suppress PIO, which is caused by rate limiting and pure time delay. Due to its architecture, the $L 1$ adaptive controller will achieve a desired response with fast adaptation. The simulation results indicate that the $L 1$ adaptive control is efficient in solving this kind of problem.

In the paper entitled "Adaptive control for a class of nonlinear system with redistributed models" by H. Ke, and J. Li, a novel multiple model adaptive controller for a class of nonlinear system in parameter-strict-feedback form is proposed. It not only improves the transient performance significantly, but also guarantees the stability of all the states of the closed-loop system. A simulation example is proposed to illustrate the effectiveness of the developed multiple model adaptive controller.

The paper entitled "Adaptive Control Allocation in the Presence of Actuator Failures" by Y. Liu and L. G. Crespo proposes a control allocation framework, where a feedback adaptive signal is designed for a group of redundant actuators and is then adaptively allocated among all group members. In the adaptive control allocation structure, cooperative 
actuators are grouped and treated as an equivalent control effector. Two adaptive control allocation algorithms are developed.

The implementation and effectiveness of the strategies proposed is demonstrated in detail using several examples.

In the paper entitled "General form of model-free control law and convergence analyzing" by $X . L i$ and $Z$. Han, the general form of model free control law is introduced and its convergence is analyzed. First, the necessity to improve the basic form of the model-free control law is explained and the functional combination method as the approach of improvement is presented. Then, a series of sufficient conditions required for the convergence are given. The analysis demonstrates that these conditions can be satisfied easily in engineering practice.

In the paper entitled "Nonlinear dynamic model-based adaptive control of a solenoid-valve system" by $D$. Lee, P. Naseradinmousavi, and C. Nataraj, a nonlinear model-based adaptive control approach is proposed for a solenoid-valve system. The challenge is that solenoids and butterfly valves have uncertainties in multiple parameters in the model, which makes it difficult for the system to adjust to the environment. These kinds of valves have different varying physical attributes such as stroke, size, and weight; uncertain parameters including inertia, damping, and torque coefficients; and various kinds of operational parameters such as pipe diameters and flow velocities. These uncertainties are further complicated because of the solenoid and butterfly valve nonlinear dynamic models. The main contribution of this research is the application of adaptive control theory and Lyapunov type stability approach to design a controller for a dynamic model of the solenoid-valve system in the presence of those uncertainties. Numerical simulation results are shown to demonstrate good performance of the proposed nonlinear model-based adaptive approach. Also shown are comparisons of the performance of the same solenoid-valve system with a nonadaptive method.

The paper entitled "Discrete model reference adaptive control system for automatic profiling machine" by P. Song, G. $K . X u$ and $X$. C. Zhao discusses that an automatic profiling machine is a system which has a high degree of parameter variation and high frequency of transient process. In this paper, a discrete model reference adaptive control system is applied on an automatic profiling machine. The results of simulation show that the adaptive control system has the desired dynamic performance.

Chengyu Cao

Lili Ma

Yunjun Xu 

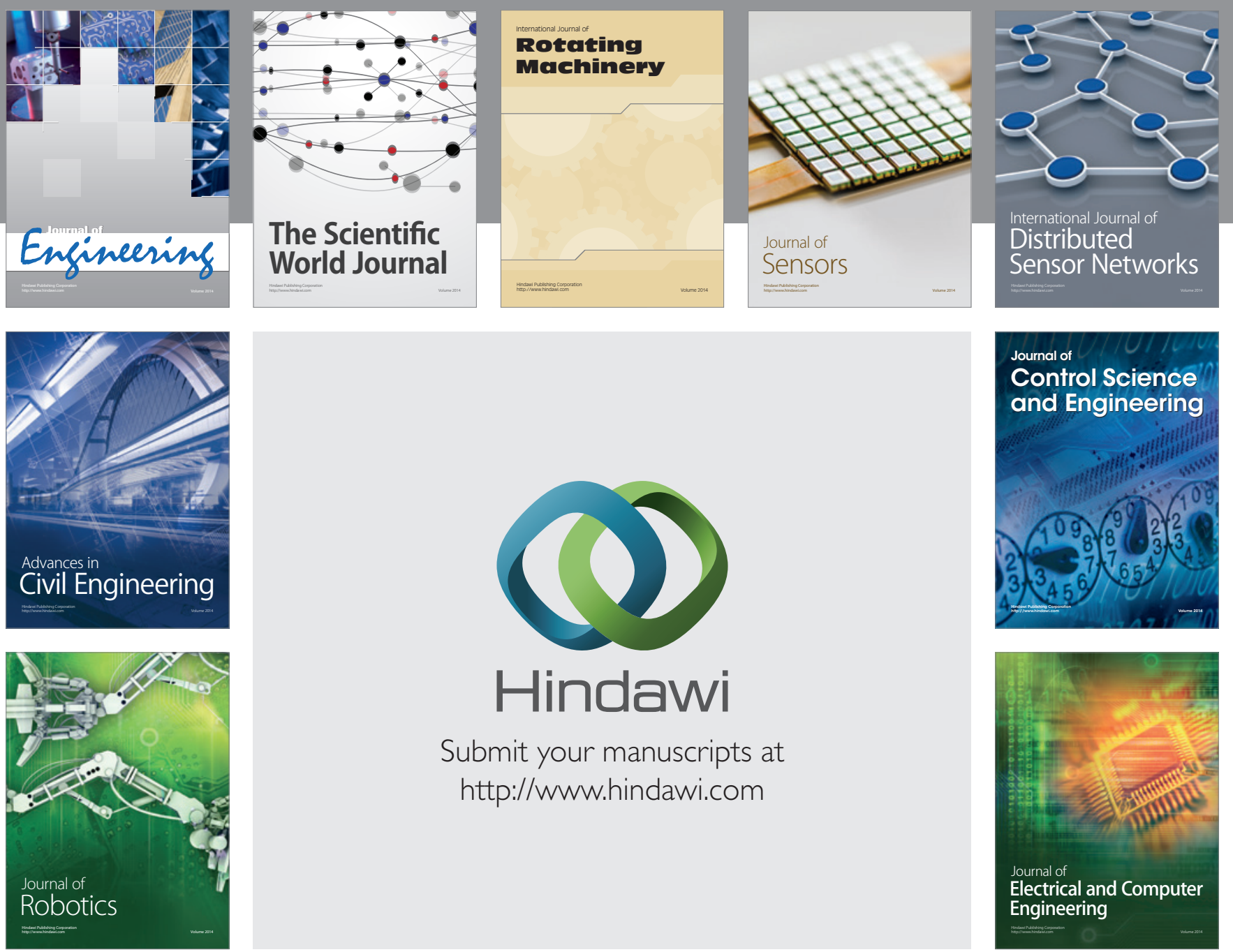

Submit your manuscripts at

http://www.hindawi.com
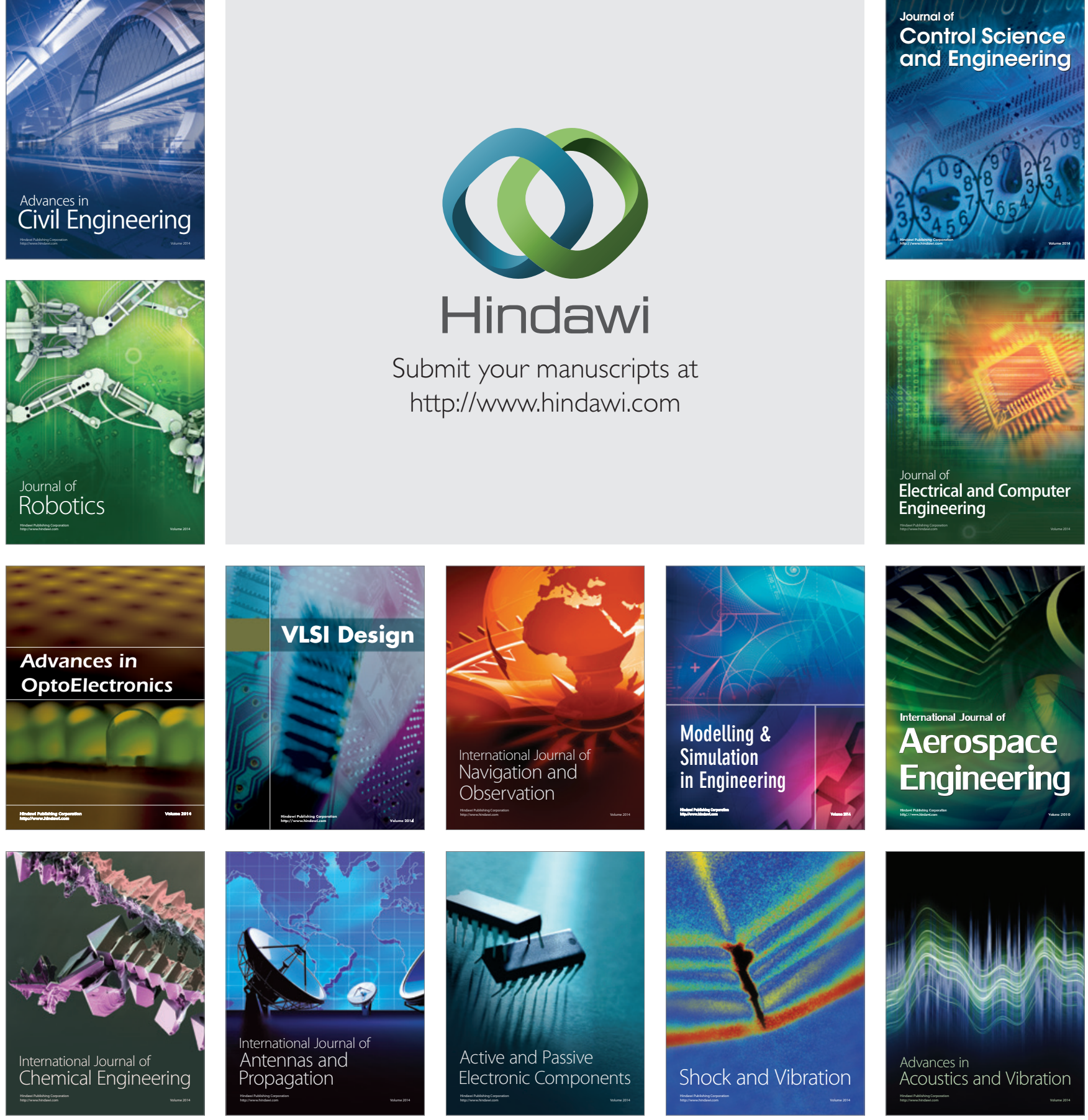